\title{
How Personal Video Navigation History can be Visualized
}

Abir Al-Hajri, Matthew Fong, Gregor Miller and Sidney Fels* Human Communication Technologies Laboratory, University of British Columbia, Vancouver, BC, Canada
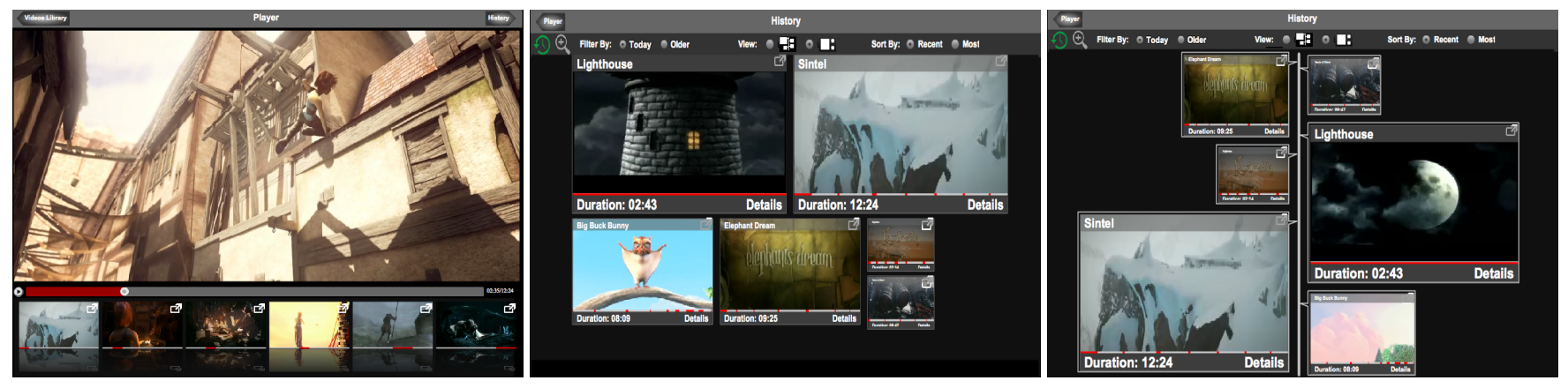

The Video History System (VHS) manages a user's personal viewing history and provides mechanisms for quick navigation. Playback is controlled with familiar tools such as play/pause/seek, and previews are arranged as a filmstrip (left). As the user watches video, the VHS records each interval viewed. The history is then visualized in one of two ways: as Video Tiles (centre) or as a Video Timeline (right). ${ }^{1}$

Video navigation histories are a simple archive that a person can use to easily find a previously viewed video interval. They may navigate to the exact location within the original video simply by clicking on the references within their history. This provides the user with a record for historical navigation and removes much of the burden of relying on memory. This history offers users multiple applications such as video chaptering, monitoring their own navigation behaviour, re-watching clips, sharing clips, video summarization, creating highlight reels and even advanced applications such as video authoring [Fong et al. 2014]. However, finding previously viewed content in a navigation history is often a difficult task due to the design, organization, and volume of information to visualize. This issue has received significant attention within the context of web browsing histories, but little attention has been paid to video navigation history.

The benefits of a navigation history come from two user behaviours. First, users do not always watch a video straight through, but may skip parts (they view only a subset of the video) - this is one of the uses of the seek mechanism, for example. (Even if users watch all videos without interruption, our interface presents a recently viewed videos list, much like popular online video services.) Second, there is an emerging behaviour of re-watching parts of video, allowing visualizations to present additional information such as view count or provide estimates of the location of affective intervals within the video. The emergence of re-watching behaviour is not obvious or necessarily intuitive; however, it has been shown that users re-watch segments of video as part of contemporary video viewing, for re-experiencing and enjoying favourite parts or to discover subtle details which were previously missed, such as with instructional videos. A personal navigation history improves the user experience for these use cases [Al-Hajri et al. 2014].

*\{abira, mfong, gregor, ssfels $\} @$ ece.ubc.ca

${ }^{1}$ Video and screenshots licenced under Creative Commons Attribution 3.0, (c) 2006 - 2012, Blender Foundation
We propose an interface (the VHS, shown in the figure above) to provide efficient access to previously viewed videos, and in particular, the intervals viewed within those videos. We accomplish this by recording a continuous video history for the user as they consume video. Navigation-level events are captured by recording high-level user actions such as seek, play, pause, and changing video. Each time a user watches a video segment, a new entry is added to the history record. To provide simple access to the history we propose two visualizations of a detailed multiple-video navigation history: Video Timeline and Video Tiles. Video Timeline extends the commonly employed list-based visualization for navigation history by using thumbnail size to indicate heuristics and occupying the full screen with a two-sided timeline. Video Tiles visualizes history items in a flow-based layout by following predefined templates based on items' heuristics and ordering, utilizing screen space more effectively at the expense of a clearer temporal location. These visualizations are easily integrated into a video viewer together or individually as demonstrated in our Video History System (VHS).

These visualizations are compared against the commonly employed list-based representation of the visited videos in a reverse chronological order (e.g. YouTube, Netflix), with ten participants tasked with sharing their previously-seen affective intervals. Our study shows that the proposed visualizations are significantly faster and are perceived as intuitive and are strongly preferred to the current method [Al-Hajri et al. 2014]. Based on these results, Video Timeline and Video Tiles provide an effective addition to video viewers to help manage the growing quantity of video. They provide users with insight into their navigation patterns, allowing them to quickly find previously-seen intervals, leading to efficient clip sharing, summarization and simpler authoring.

\section{References}

Al-Hajri, A., Miller, G., Fong, M., AND Fels, S. 2014. Visualization of personal history for video navigation. In Proceedings of the Conference on Human Factors in Computing Systems, ACM, CHI '14, 1187-1196.

Fong, M., Al Hajri, A., Miller, G., AND Fels, S. 2014. Casual authoring using a video navigation history. In Proceedings of the Graphics Interface Conference, CIPS, 109-114. 